\begin{tabular}{|c|c|c|c|c|c|}
\hline Cuadernos I. Geográfica & 15 & $1-2$ & $87-98$ & Logroño & 1989 \\
\hline
\end{tabular}

\title{
ORGANIZACION ESPACIAL DE LAS TEMPERATURAS EN LA CIUDAD DE LOGROÑO
}

\author{
José M. García Ruiz* \\ Luis M. Ortigosa Izquierdo** \\ José Arnáez Vadillo** \\ Amelia Gómez Villar***
}

\begin{abstract}
RESUMEN
Mediante un muestreo llevado a cabo a las 6,15 y 23 horas a lo largo de 1987, se han medido las temperaturas en 23 puntos (ejes $S-N / E-W$ ) de la ciudad de Logroño. El muestreo pretendía confirmar la existencia de una «isla de calor» en el espacio urbano y contrastar el funcionamiento térmico entre el centro y la periferia. El tratamiento estadístico de los datos permite hablar efectivamente de un clima urbano, aunque con contrastes térmicos muy pequeños. Se ha comporbado que, sobre todo a las 23 horas en verano, el centro de la ciudad funciona como una auténtica «isla de calor», situada $3 .^{\circ}$ por encima de los alrededores.
\end{abstract}

\section{SUMMARY}

By a sampling realized to the 6,15 and 23 hours during 1987, we have measured the temperatures on 23 points (axis $S-N / E-W$ ) of Logrono city. The sampling seeks to confirm the existence of $a$ "heat island" in the city and to contrast the thermic functioning between centre and outskirts. The statistical processing of the data allows to attribute to Logroño an urban climate. We have confirmed that, principally at the 23 hours in summer, the city center functions as a "heat island» with temperatures of $3^{\circ} \mathrm{C}$ higher than those of the outskirts.

PALABRAS CLAVE: temperaturas, isla de calor, clima urbano, Logroño.

KEY WORDS: temperature, heat island, urban climate, Logroño.

* Instituto Pirenaico de Ecología. Apartado 64, JACA, (Huesca).

** Departamento de Geografía. Colegio Universitario de La Rioja. Logroño 


\section{GARCIA RUIZ, ORTIGOSA, ARNAEZ Y GOMEZ}

Desde los estudios de HOWARD de principios del siglo XIX (Vid. LEE 1984), se tiene constancia precisa de la notable alteración climática que representan los grandes centros urbanos e industriales. En los últimos treinta años esa evidencia ha cobrado más fuerza dado el proceso de urbanización que afecta a la práctica totalidad de nuestro planeta. Esto explica la gran importancia que, tanto desde el campo de la climatología como del de la geografía urbana, están alcanzando los estudios sobre microclimas urbanos. Debe tenerse en cuenta que el estudio del clima de la ciudad tiene un doble componente geográfico: por un lado el núcleo urbano presenta unas características térmicas $-\mathrm{y}$ a veces también pluviométricas- diferentes del entorno rural. Ello es así porque la ciudad genera calor, cuenta en su atmósfera con mayor proporción de partículas en suspensión y elementos contaminantes, no tiene las mismas posibilidades de evaporación y autorregulación térmica y está formada por materiales de baja conductividad térmica, que acumulan mucho calor en superficie. Por otro lado, el interior de la ciudad no se comporta homogéneamente, pues la gran variedad de formas y orientaciones genera contrastes en pequeños espacios e individualiza áreas de funcionamiento diferente. El lector encontrará en el reciente trabajo de LEE (1984) amplia referencia sobre las líneas de trabajo más desarrolladas en el campo de la climatología urbana, así como una extensa lista bibliográfica.

Es importante tener en cuenta que la práctica totalidad de los estudios se han realizado en los mayores centros urbanos y en los más importantes complejos industriales, porque es en ellos donde mejor puede apreciarse la influencia climática de la ciudad y su contraste con el medio rural más próximo. En España, por ejemplo, donde este tipo de aproximaciones cuenta con escasa tradición, ha sido Madrid la ciudad que ha atraído más la atención de los geógrafos, primero con una perspectiva temporal (LOPEZ GOMEZ, 1961) y, más recientemente, desde un punto de vista espacial (LOPEZ GOMEZ y FERNANDEZ GARCIA, 1984; LOPEZ GOMEZ, dir., 1988). En estos últimos trabajos se insiste especialmente en la «isla de calor» del centro urbano, similar a la de otras ciudades del mundo, aunque complicada por la topografia de Madrid. En algún caso se alude al efecto del casco urbano madrileño sobre la intensidad del viento (FERNANDEZ GARCIA, 1982). Por el contrario, son pocos los estudios llevados a cabo en ciudades medianas o pequeñas, salvo que se añada otra característica como la accidentada topografía (FALLOT y BUMAN, 1986). No obstante LOPEZ GOMEZ y FERNANDEZ GARCIA (1984) han comprobado que en las barriadas y núcleos periféricos de Madrid, algunos con más de 100.000 habitantes, se producen efectos climáticos similares a los de las grandes ciudades, con islas de calor bien definidas (a veces hasta con $4^{\circ}$ $\mathrm{C}$ más que en sus alrededores). Este trabajo se centra precisamente en una ciudad media, como es Logroño, con el fin de comprobar si verdaderamente cabe hablar de un "clima urbano» y de su correspondiente isla de calor, a la vez que se presentan algunos rasgos de la organización espacial de las temperaturas.

\section{LA CIUDAD DE LOGROÑO}

Situada a orillas del río Ebro, muy cerca de la confluencia del río Iregua con este último, se localiza a $384 \mathrm{~m}$. de altitud, sobre terrazas bajas. Se asienta sobre una topografia muy suave, sin accidentes de relieve, aunque a poca distancia hacia el Sur existen restos de antiguos niveles cuaternarios, cuya influencia en el clima de la ciudad parece más bien marginal. El crecimiento urbano se ha desarrollado a partir de un pequeño núcleo medieval surgido en torno al Camino de Santiago, si bien no cabe hablar de verdadero aumento espacial hasta principios del siglo XX. Ese crecimiento siempre ha tenido lugar hacia el 


\section{TEMPERATURAS EN LOGROÑO}

Oeste, Sur y Este, en sucesivos ensanches, siguiendo las salidas hacia Burgos, Soria y Zaragoza. El plano muestra una organización bastante regular en cuadrícula, con una vía principal -Gran Vía -Jorge Vigón- en sentido W-E y otra -Vara del Rey-Avda. de Madrid- en sentido N-S. La ausencia de grandes espacios verdes es bien conocida y sólo cabe destacar la presencia de El Espolón como relativamente amplio espacio abierto en el interior de la ciudad (vid. MUGA, 1980). En síntesis, Logroño es una ciudad de tamaño medio, con 111.000 habitantes (1981); su núcleo urbano se extiende a lo largo de 3,4 x $1,8 \mathrm{Km}$. de longitud en sus principales ejes.

Con una precipitación media anual de $451 \mathrm{~mm}$., el mes de mayor pluviosidad es junio, seguido de mayo y septiembre, con mínimos en verano e invierno. La temperatura media anual es de $13.3^{\circ} \mathrm{C}$, con un pico en julio de $21.8^{\circ} \mathrm{C}$ de media y agosto con $21.7^{\circ} \mathrm{C}$ y mínimo en enero con $5.5^{\circ} \mathrm{C}$. Se trata, por lo tanto, de un clima mediterráneo continentalizado, que queda a medio camino de los ambientes más frescos y húmedos de La Rioja Alta y de las áreas más claramente mediterráneas de La Rioja Baja (MARTIN RANZ y GARCIA-RUIZ, 1984 y 1988).

\section{METODOS}

Se seleccionaron 23 puntos de muestreo distribuidos por toda la ciudad y en sus alrededores más próximos, siguiendo un eje $\mathrm{E}-\mathrm{W}$ y otro eje $\mathrm{S}-\mathrm{N}$ (figura 1). En cada punto las temperaturas se midieron mediante un termómetro digital Crison el primer miércoles de cada mes, a las 6,15 y 23 horas, durante el año 1987. Más tarde se incorporó al muestreo un higrómetro digital cuyos resultados no se han utilizado en este trabajo.

Con los datos recogidos se elaboró una matriz de 23 filas y 34 columnas. Se desecharon dos muestreos por no estar completos en todos los puntos. Posteriormente se calcularon las temperaturas medias de cada punto de muestreo, para el total de datos y distinguiendo entre invierno y verano y entre situación anticiclónica y ciclónica. En este trabajo presentamos un avance de los resultados más significativos por medio de los correspondientes perfiles térmicos.

Con el fin de confirmar o no la existencia de una isla de calor en el espacio urbano y de contrastar el funcionamiento térmico entre centro y periferia se trataron los datos estadísticamente mediante un cluster completo y mediante un análisis de componentes principales (PCA), tratamientos estadísticos que permiten discriminar conjuntos de datos de acuerdo con su comportamiento global y similaridad multivariable.

\section{RESULTADOS}

Las variaciones térmicas observadas están supeditadas en primer lugar a la sucesión de los tipos de tiempo a lo largo del año y, en segundo lugar, a la hora en que se efectuó el muestreo. Así en invierno y a primeras horas de la mañana se han observado las más bajas temperaturas (por debajo de $0^{\circ}$ en todos los puntos urbanos y periurbanos). Contrariamente, en los meses veraniegos y a las $15 \mathrm{~h}$. de la tarde, se superaban los $30^{\circ}$ de temperatura en cualquier punto de la ciudad. Estas variaciones quedan explicadas por el régimen climático 


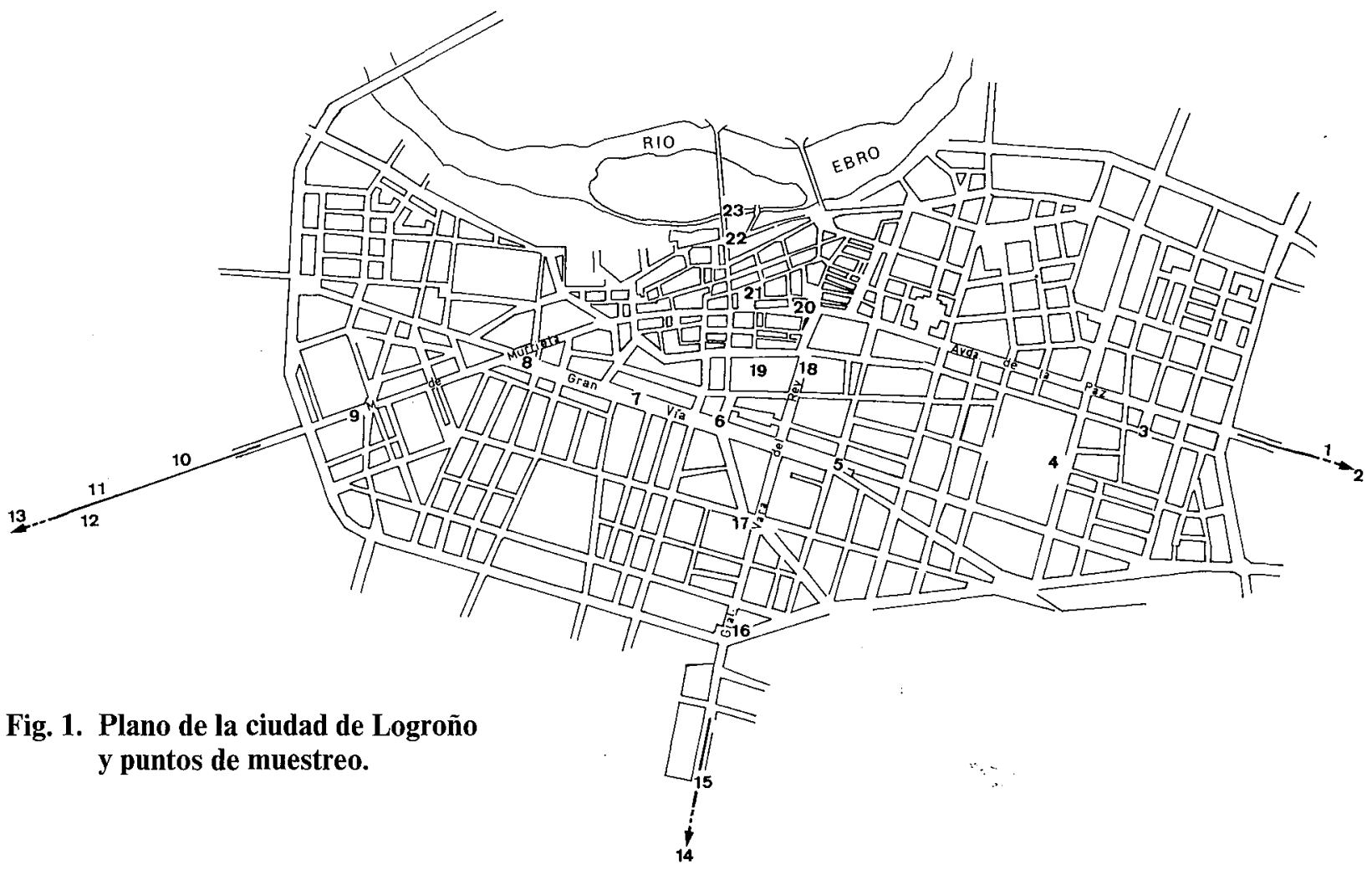

Fig. 2 DENDROGRAMA DEL CLUSTER (Complete linkage)

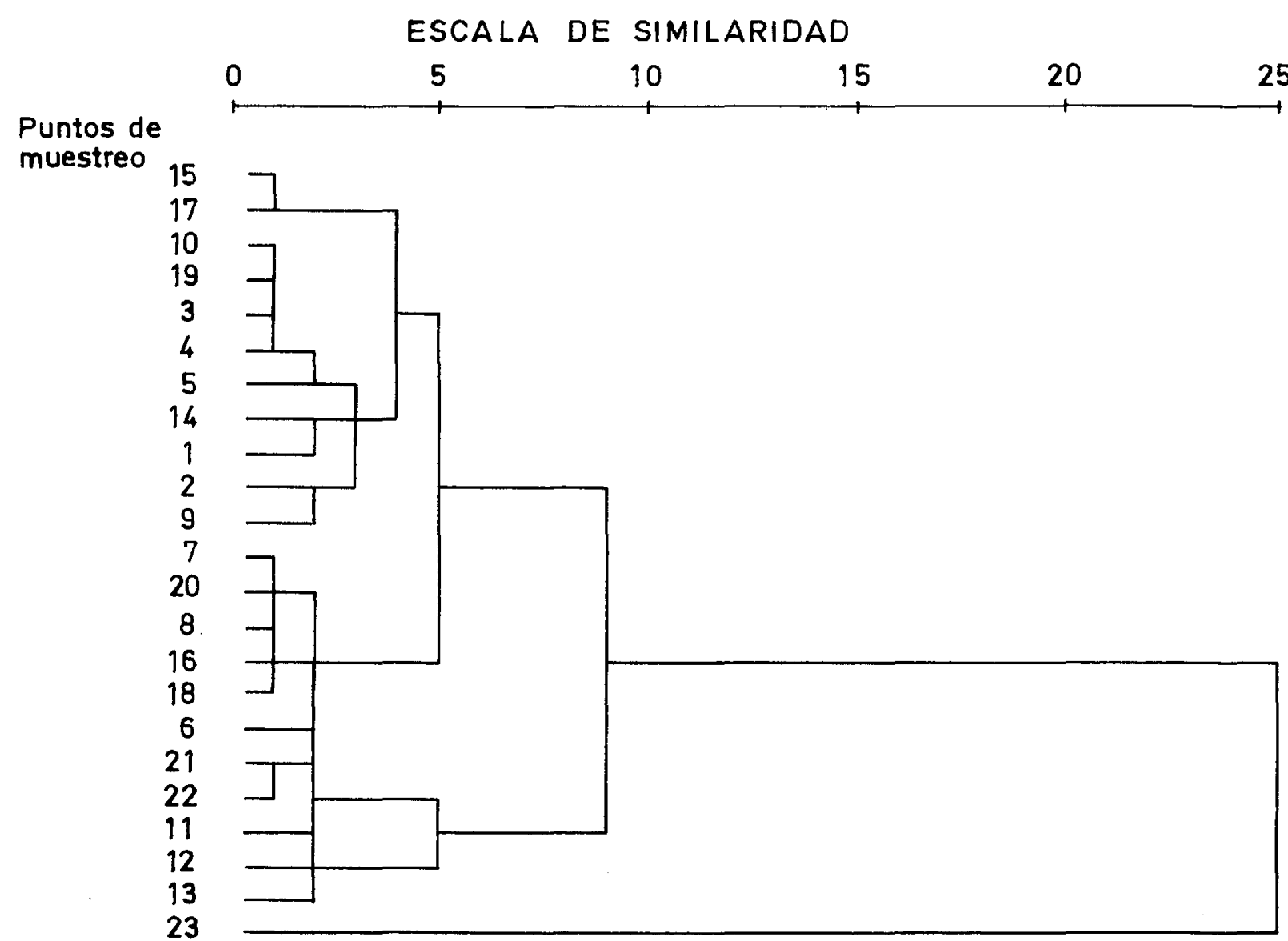




\section{TEMPERATURAS EN LOGROÑO}

del entorno, y carecen de interés desde la perspectiva del microclima urbano. En este sentido, resulta más importante descubrir las diferencias y similitudes entre los diferentes sectores de la ciudad.

Utilizando los valores térmicos en los diversos puntos de muestreo a lo largo del año, realizamos un procedimiento de cluster (Complete linkage) que discrimina a grandes rasgos dos tipos de sectores. Por un lado están los puntos correspondientes al centro de la ciudad, y, por otro, los situados en la periferia (todos los que se localizan en la circunvalación, más el alto de la Grajera y el Puente de Hierro sobre el río Ebro). Mediante esta técnica estadística multivariable ha resultado evidente que el núcleo interior de Logroño cuenta con un funcionamiento térmico diferente. El dendrograma resultante de los agrupamientos del cluster así lo demuestra (figura 2).

Con la misma matriz de datos y buscando nuevas interpretaciones del comportamiento térmico de los puntos de muestreo, hemos realizado un análisis factorial (vía componentes principales, PCA), cuyo objetivo es resumir la varianza original en unas nuevas componentes, tantas como variables, que acumulan progresivamente menor varianza (tabla 1). La primera componente absorbe la mayor parte de la varianza acumulada $(99,3 \%)$, y recoge el efecto de comunalidad que existe entre todos los puntos de muestreo en función de los valores térmicos mensuales. Ello se debe a que entre las variables utilizadas existe un grado muy elevado de autocorrelación (siempre superior a 0.97, con un nivel de significación del 0.999), pues los valores de cada variable dependen esencialmente del tipo de tiempo en cada momento de muestreo. En este caso, la segunda componente, aunque absorbe una pequeña proporción de la varianza, adquiere gran valor para explicar la variabilidad de la muestra. $\mathrm{Y}$, en efecto, esa componente separa a grandes rasgos dos conjuntos de puntos de muestreo.

a) Estatua del Labrador, Gran Vía, Cruce Vara del Rey-Pérez Galdós, Centro del Espolón y Catedral de La Redonda.

b) Cuartel de la Guardia Civil, Tramo final de Avda. Burgos, Circunvalación, comienzo de Carretera de Soria, Puente de Hierro y Alto de la Grajera.

Tabla 1. Varianza de las componentes en el análisis factorial (PCA)

\section{Component Number}

Percent of Variance

99.29333

.16608

.11159

.09589

.07460

\section{Cumulative Percentage}

99.29333

99.45941

99.57100

99.66689

99.74149 


\section{PESO DE LAS VARIABLES EN LAS TRES PRIMERAS COMPONENTES DEL ANALISIS FACTORIAL (PCA)}

\section{FACTOR 1}

$\begin{array}{ll}\text { VAR 1 } & .00624 \\ \text { VAR 2 } & .99647 \\ \text { VAR 3 } & .99735 \\ \text { VAR 4 } & .99661 \\ \text { VAR 5 } & .99707 \\ \text { VAR 6 } & .99610 \\ \text { VAR 7 } & .99825 \\ \text { VAR 8 } & .99711 \\ \text { VAR 9 } & .99650 \\ \text { VAR 10 } & .99754 \\ \text { VAR 11 } & .99583 \\ \text { VAR 12 } & .99574 \\ \text { VAR 13 } & .99359 \\ \text { VAR 14 } & .99789 \\ \text { VAR 15 } & .99782 \\ \text { VAR 16 } & .99688 \\ \text { VAR 17 } & .99805 \\ \text { VAR 18 } & .99660 \\ \text { VAR 19 } & .99775 \\ \text { VAR 20 } & .99761 \\ \text { VAR 21 } & .99528 \\ \text { VAR 22 } & .99644 \\ \text { VAR 23 } & .98985\end{array}$

\section{FACTOR 2}

$-.1908$

$-.02972$

.00520

$-.00565$

$-.03344$

$-.05446$

$-.03224$

$-.04180$

.02967

$-.00730$

.03133

.05646

.02953

$-.00194$

$-.00331$

$-.04206$

.00766

$-.04777$

$-.01209$

$-.03725$

.05795

.03356

.11792

\section{FACTOR 3}

.04155

.00496

.01811

.03025

.02361

.04115

.01237

.01481

.01810

$-.02931$

$-.04133$

.04474

.06703

.01528

$-.02830$

$-.01897$

$-.03995$

$-.03888$

$-.04675$

$-.01609$

$-.04910$

$-.03636$

.01350

Con pequeñas diferencias, este esquema reproduce lo obtenido mediante el cluster y se corrobora con el trazado de los perfiles de temperaturas medias (figura 3). Los dos perfiles ( $\mathrm{S}-\mathrm{N}$ y $\mathrm{E}-\mathrm{W}$ ) muestran un claro incremento térmico desde la periferia hacia el interior de la ciudad, alcanzándose el máximo en Jorge Vigón-Estatua del Labrador y el mínimo en el Alto de la Grajera y en la salida hacia Zaragoza. Interesa señalar, no obstante, que en el perfil S-N se produce una marcada inflexión en el centro de El Espolón, seguramente por la presencia de arbolado y fuente, con la consiguiente moderación de las temperaturas; por otro lado, al tratarse de un punto alejado de fachadas, no recibe radiación reflejada con la misma intensidad que otros puntos de muestreo. En ese mismo perfil, una segunda inflexión de menor entidad se produce a la altura de la Catedral, en pleno casco antiguo, seguramente porque la estrechez de las calles reduce la insolación. En todo caso, las diferencias entre unos puntos y otros, medidas en grados, son muy pequeñas: $1.6^{\circ} \mathrm{C}$ en el perfil $\mathrm{E}-\mathrm{W}$ y $0.6^{\circ} \mathrm{C}$ en el perfil $\mathrm{S}-\mathrm{N}$ (ver tabla 2).

La figura 4 refleja el coeficiente de variación de las temperaturas en cada uno de los puntos de muestreo, como una medida de dispersión de los datos. Esa variabilidad parece ser mayor en la periferia que en el centro, lo que alude al conocido «efecto invernadero» en el interior de las ciudades, aunque sea de forma muy matizada. En todo caso, la menor variación térmica se produce en El Espolón, confirmando la influencia moderadora de la cubierta arbórea. 


\section{TEMPERATURAS EN LOGROÑO}

La isla de calor y las diferencias espaciales se aprecian mejor en la figura 5 , en la que representamos los perfiles térmicos a las 6,15 y 23 horas, distinguiendo entre invierno y verano. En cada una de las curvas se incluyen los dos perfiles, como si se tratase de un muestreo espacialmente continuo, para simplificar su representación. La isla de calor se reproduce perfectamente a las 6 y a las 23 horas en verano; es también apreciable, aunque con notables dificultades, a esas mismas horas en invierno; pero es inexistente a las 15 horas, tanto en invierno como en verano. La comparación de los dos gráficos demuestra, en todo caso, que es en verano cuando más claramente aparece un «clima urbano» en la ciudad de Logroño. Ello no deja de ser lógico, pues los materiales que componen básicamente los edificios y calles favorecen la acumulación de calor y la irradiación, a la vez que dificultan la evaporación por ausencia de agua en superficie. Por el contrario, en invierno los efectos de las calefacciones son poco apreciables. A su vez, las curvas correspondientes a las 15 horas, muestran una gran irregularidad espacial y carecen de pautas lógicas. Se trata del momento de máxima insolación y las temperaturas de cada punto dependen en su variabilidad de muchos factores, entre los que la proximidad o alejamiento de las paredes no debe ser poco importante, así como la radiación recibida en el momento del muestreo sumada a la de las horas precedentes. En definitiva, es un momento en el que la variación de las temperaturas se halla más relacionada con la distribución de las sombras y las orientaciones respecto al sol que con la posición de cada punto de muestreo en el interior de la ciudad.

Tabla 2. Puntos de muestreo en Logroño

\section{VARIABLE}

1. Ctra. Zaragoza

2. Ctra. Zaragoza-Entrada Logroño

3. Residencia Sanit.

4. Plza. Las Chirivitas

5. Estatua Labrador

6. Gran Vía-Ayda. La Rioja

7. Centro Gran Vía

8. Gran Vía-Murrieta

9. Cuartel G. Civil

10. Avda. Burgos

11. Avda. Burgos-Barrio Yagüe

12. Avda. Burgos Circunvalación

13. Alto de la Grajera

14. Ctra. Soria

15. Ctra. Soria Circunvalación

16. Vara de Rey-Huesca

17. Vara de Rey-Pérez Galdós

18. Espolón

19. Espolón-Centro

20. Portales-M. Carmen

21. Catedral Redonda

22. Puente Hierro

23. Puente Hierro
Media

14.2

14.4

15.1

14.8

15.2

14.8

14.9

14.8

14.8

14.2

13.8

13.9

13.6

14.1

14.4

14.5

14.7

14.8

14.2

14.8

14.5

14.9

14.7
Std. Des. Minimum Maximum

87.72

82.01

89.65

86.75

86.37

84.02

81.87

80.48

82.68

76.27

80.80

79.26

83.61

81.89
323

348

328

319

$-12$

$-12$

$-19$

$-27$

$-22$

$-18$

$-34$

334

360

327

$-25$

$-18$

$-9$

$-16$

$-16$

$-9$

$-17$

$-11$

$-14$

315

328

322

327

290

322

310

333

325
312

317

336

336

324

318

316

N. Label

34

34

34

34

34

34

34

34

34

34

34

34

34

34 
Fig. 3 VARIACIONES TERMICAS DE LA CIUDAD DE LOGROÑO $\circ \mathrm{C}$

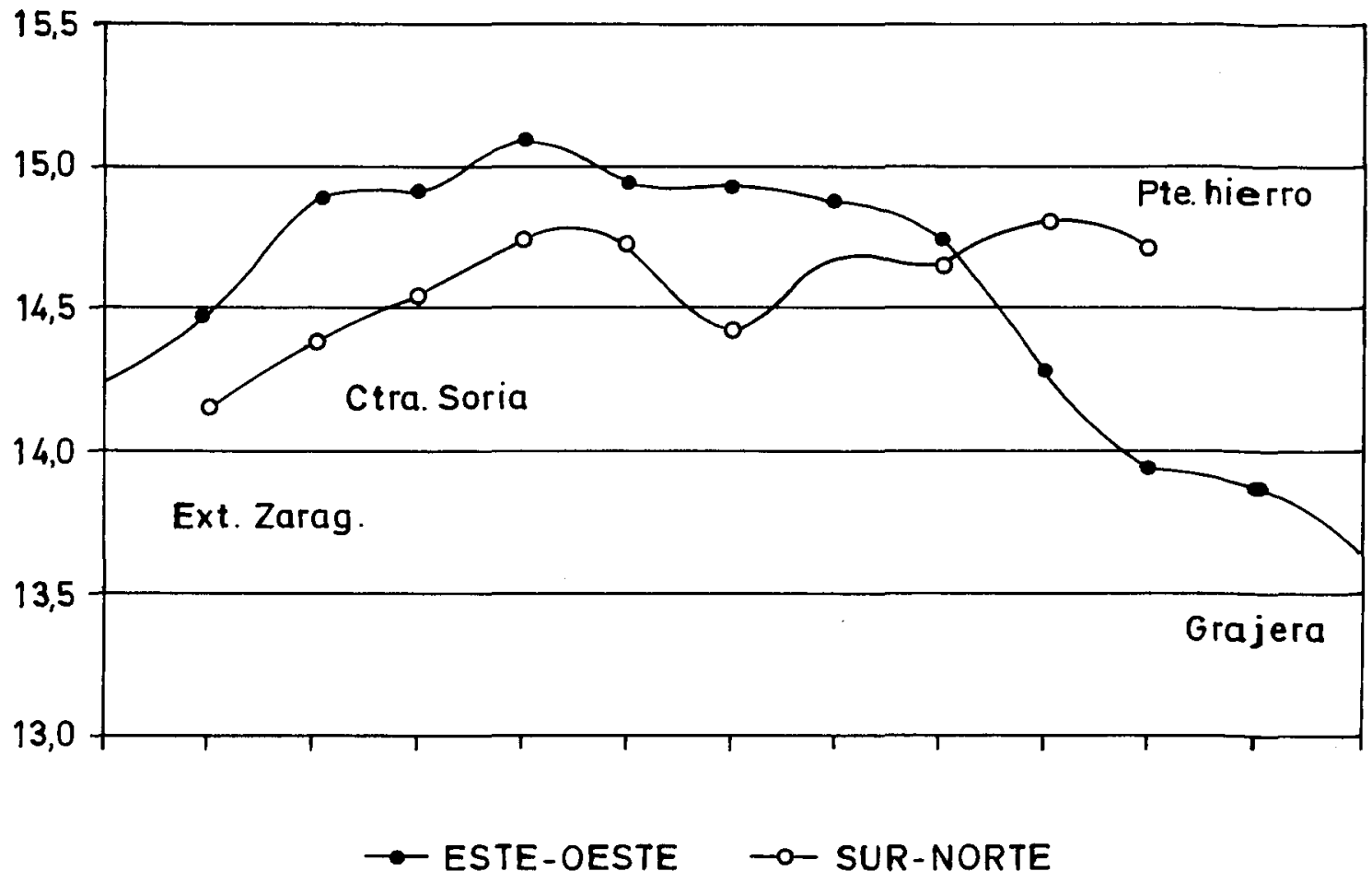

Fig. 4 COEF. VARIACION TERMICO DE LA CIUDAD DE LOGROÑO

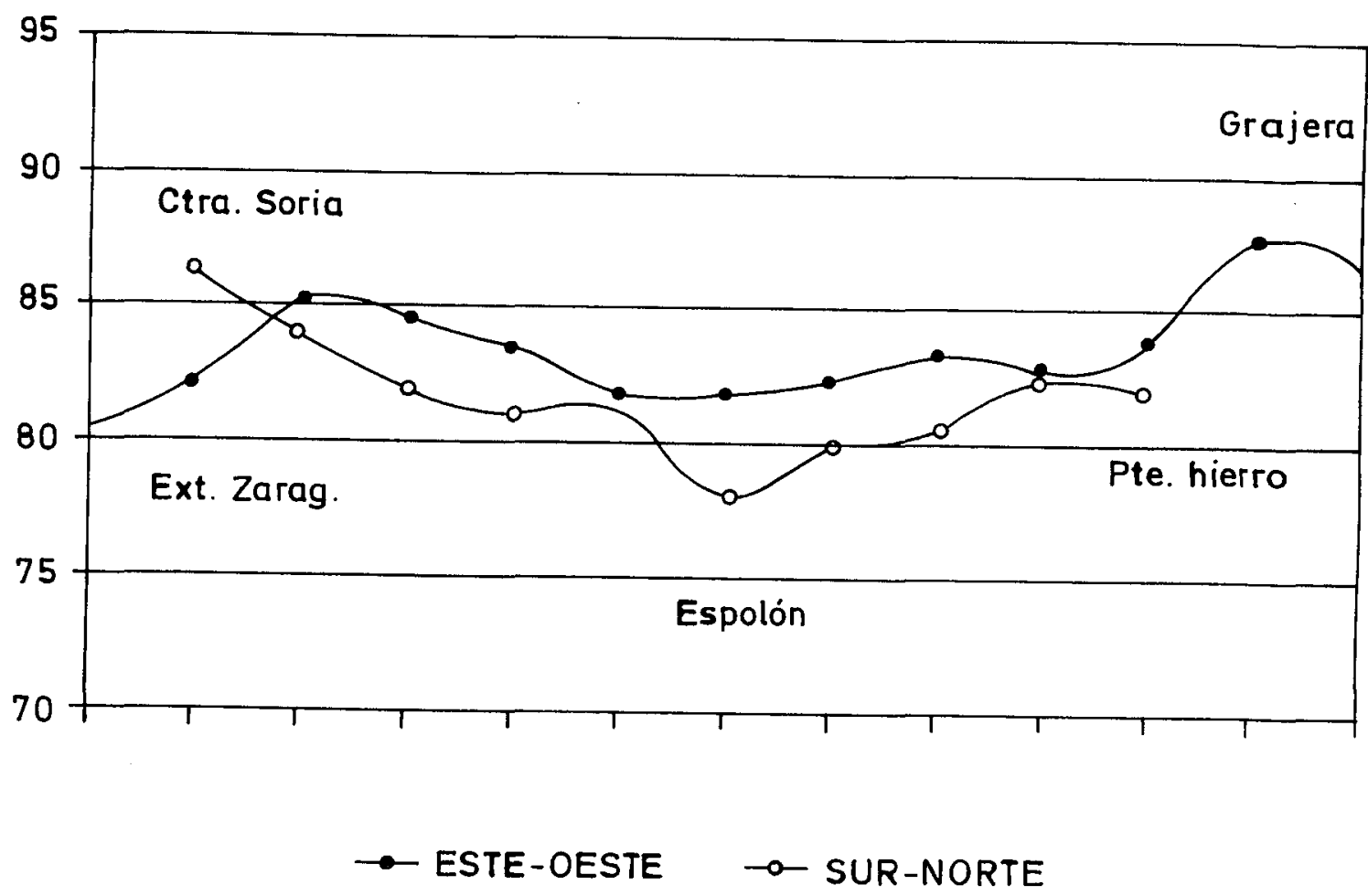




\section{TEMPERATURAS EN LOGROÑO}

Otra cuestión interesa destacar al comparar las temperaturas de invierno y verano. En invierno las temperaturas correspondientes a las 6 y a las 23 horas están muy próximas entre sí, lo que no sucede en verano. Esa diferencia se explica porque en invierno a las 23 horas ya se ha producido la irradiación del calor acumulado durante el día, habida cuenta del tiempo transcurrido desde la puesta de sol; de ahí que la temperatura se aproxime a la del amanecer. Por el contrario, en verano la ciudad mantiene a las 23 horas gran parte de la energía recibida, que seguirá perdiendo durante la noche hasta alcanzar el mínimo a primeras horas de la madrugada. A este respecto resulta aleccionadora la figura 6, en la que se reflejan las temperaturas de verano e invierno a las 23 horas, con sus correspondientes coeficientes de variación. La isla de calor se dibuja perfectamente en verano, incluida la pequeña inflexión de El Espolón, con diferencias térmicas que oscilan en torno a $2.5^{\circ} \mathrm{C}$ entre centro y periferia. En ese momento, es cuando mejor se manifiesta el diferente comportamiento térmico entre la ciudad y sus alrededores. Los materiales con que está construida la ciudad acumulan mucho calor en superficie por su baja conductividad, de manera que a primeras horas de la noche se produce una liberación de energía que prolonga parcialmente las altas temperaturas del día; en la periferia el descenso de la temperatura tras la puesta de sol es más rápida al no existir esos acumuladores de energía que son el asfalto y las paredes. Por esa misma razón, la isla de calor es menos prolongada $y$, además, sus efectos se hallan ya muy disminuidos varias horas después de la puesta de sol. Aun así se produce un notable contrastre entre centro y periferia, sobre todo en el perfil E-W. El perfil S-N es menos evidente, sobre todo en invierno por el especial comportamiento del punto de muestreo más septentrional, correspondiente al Puente de Hierro sobre el río Ebro. En este último caso las temperaturas son más frescas que en el resto de la ciudad durante el verano, por el efecto refrescante de la evaporación; pero en invierno las temperaturas son más templadas -aunque sólo ligeramente- por la mayor tibieza de las aguas corrientes.

\section{DISCUSION}

Las figuras 3 y 6 demuestran claramente el incremento medio de temperatura que se produce desde la periferia hacia el interior de la ciudad de Logroño. En ese sentido cabe hablar, pues, de un "clima urbano», aunque los contrastes térmicos son muy pequeños. Pero la sola presencia de un entramado de calles y bloques, con su peculiar conductividad, basta para alterar el balance de energía y el ritmo de radiación-irradiación. Los materiales que componen básicamente las estructuras urbanas poseen una elevada resistividad térmica y por ello acumulan el calor en una capa muy superficial, lo que les permite restituir pronto esa energía en la atmósfera en la primeras horas de la noche. Por ello, aunque los perfiles térmicos medios son muy expresivos, aún lo es más el correspondiente a las 23 horas de verano, momento en el que el centro de la ciudad forma una auténtica «isla de calor», situada casi $3^{\circ} \mathrm{C}$ por encima de los alrededores. Por el contrario, a las tres de la tarde esa isla es más dificil de identificar por la gran importancia que adquiere en ese momento la localización del punto de muestreo (en área insolada, cerca o no de paredes). Conviene señalar que un fenómeno similar ha sido constatado en Madrid (LOPEZ GOMEZ y FERNANDO GARCIA, 1984), donde la isla de calor se aprecia en el momento de las temperaturas mínimas. Según se ha podido demostrar en Madrid en el momento de las máximas el factor condicionante de la temperatura de cada punto no es la distancia al centro, sino el emplazamiento de los observatorios. 
Fig. 5 PERFILES TERMICOS MEDIOS EN INVIERNO Y VERANO

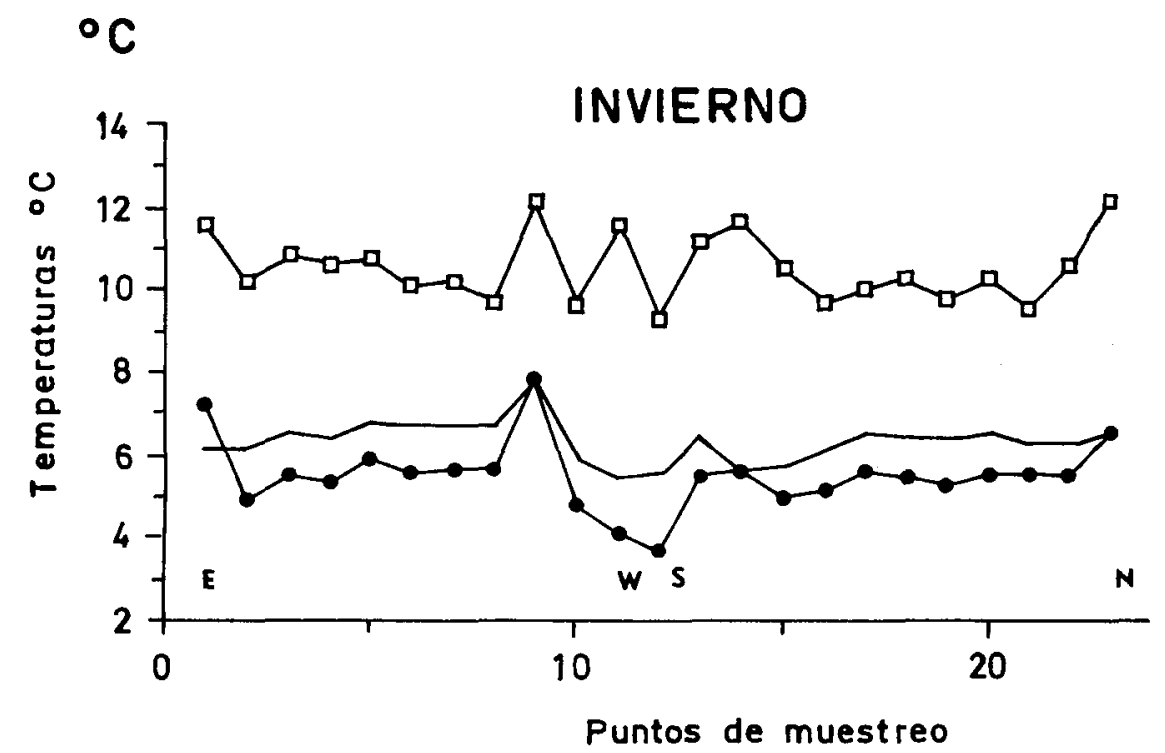

$\rightarrow$ Invierno $15 \mathrm{~h}$

$\rightarrow$ Invierno $6 \mathrm{~h}$

- Invierno $23 \mathrm{~h}$

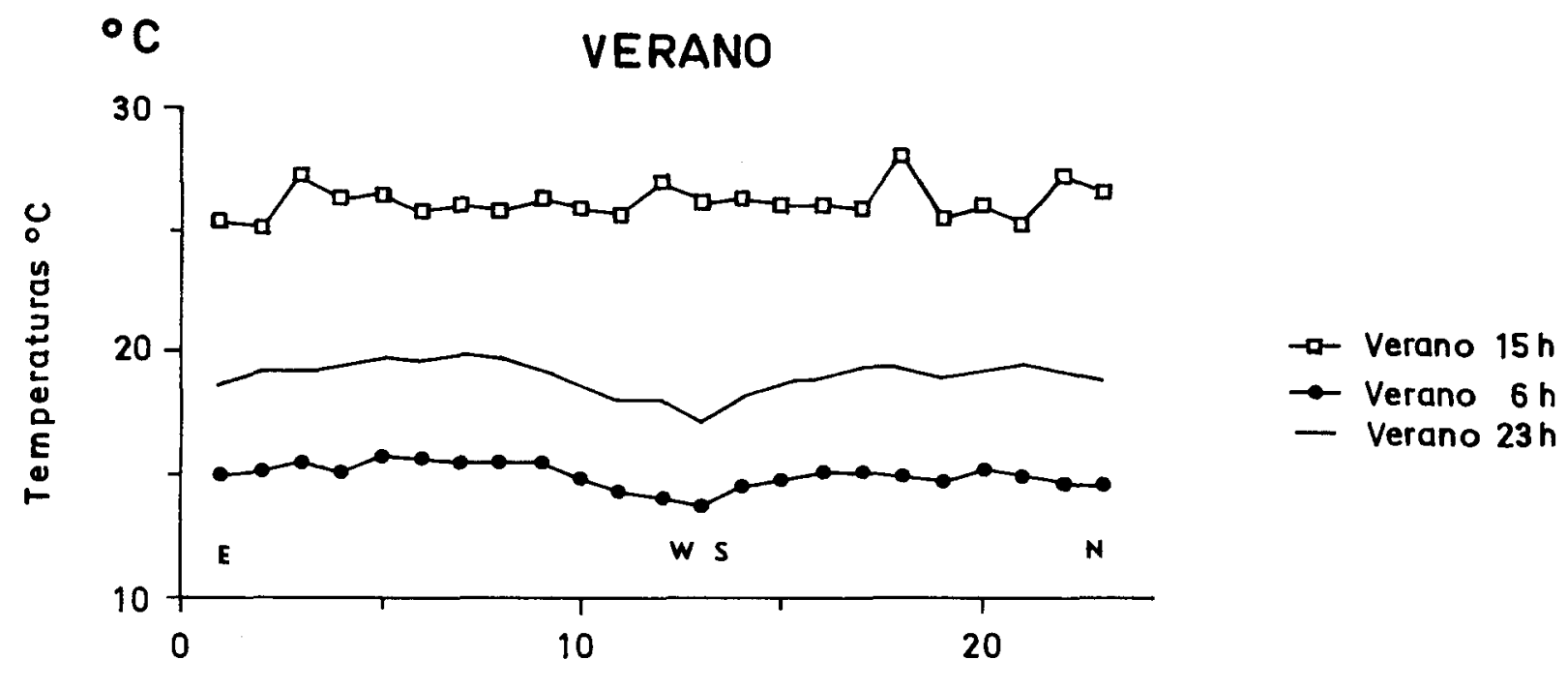

Puntos de muestreo 
TEMPERATURAS EN LOGROÑO

Fig. 6 PERFILES TERMICOS MEDIOS, A LAS 23 HORAS
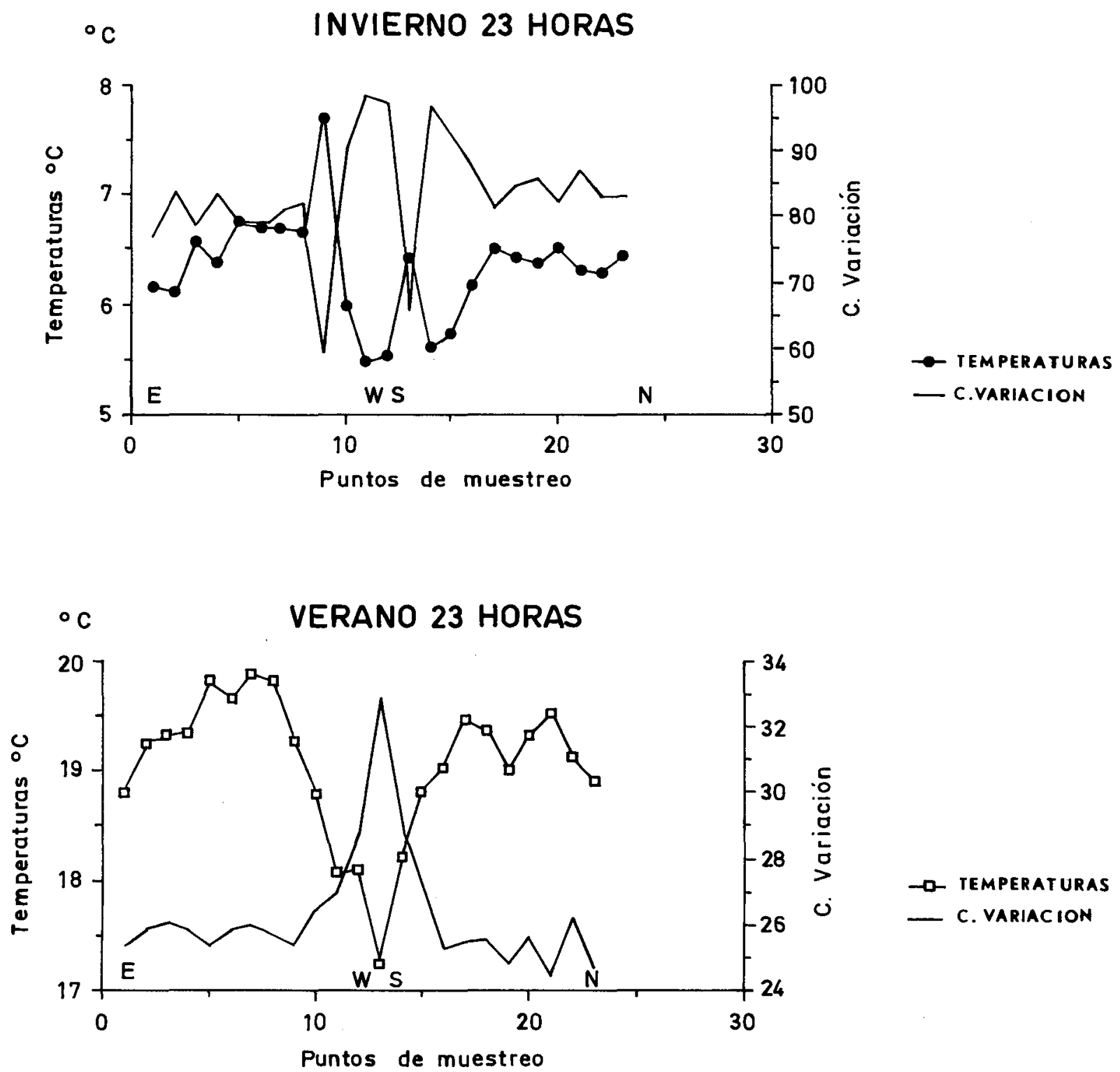


\section{GARCIA RUIZ, ORTIGOSA, ARNAEZ Y GOMEZ}

Aunque los contrastes térmicos en el interior de Logroño son pequeños debido sobre todo a su limitación espacial y a la ausencia de accidentes topográficos, el muestreo realizado ha sido sensible a algunos rasgos de su estructura urbana. Así, el parque de El Espolón, aunque muy asfaltado, se comporta como un sector ligeramente más fresco que sus alrededores; y lo mismo, aunque en menor medida, parece suceder con las calles del casco antiguo, cerca de la Catedral de La Redonda, al ser más estrechas y sombreadas. E1 río Ebro, con su notable caudal hídrico funciona como un pequeño regulador, aunque sus efectos se ciñen a sus más inmediatas proximidades.

\section{BIBLIOGRAFIA}

Fallot, J.M. et Buman, A.M. 1986. Mesures de l'îlot de chaleur urbain dans le cadre d'une petite ville à topographie tourmentée: le cas de Fribourg (Suisse). Freiburger Geographische Hefte, 26: 20-29.

LOPEz Gomez, A., director, 1988. El clima urbano de Madrid: la isla de calor. Instituto de Economía y Geografía Aplicadas, C.S.I.C., 199 pp., Madrid.

Lopez Gomez, A., 1961. ¿Está cambiando el clima de Madrid? Estudios Geográficos, 84-85: 575-590.

Lopez Gomez, A. y Fernandez Garcia, F., 1984. La isla de calor en Madrid. Avance de un estudio de clima urbano. Estudios Geográficos, 174: 5-34.

Fernandez Garcia, F., 1982. La intensidad del viento en Madrid y sus alrededores. Estudios Geográficos, 166: 17-32.

LeE, D.O., 1984. Urban climates. Progress in Physical Geography, 8(1): 1-31.

Martin Ranz, M.C. y Garcia-Ruiz, J.M., 1984. Los rios de La Rioja. Introducción al estudio de su régimen. Instituto de Estudios Riojanos, 66 pp., Logroño.

Martin Ranz, M.C. y Garcia-Ruzz, J.M., 1988. El clima y los recursos hídricos superficiales de La Rioja. El Campo, 110: 20-24.

Muga, M., 1980. La utilización de los espacios verdes en la ciudad de Logroño. Cuadernos de Investigación Geográfica, 6: 71-84. 